\title{
Emergent Self-Organized Complex Network Topology out of Stability Constraints
}

\author{
Juan I. Perotti, ${ }^{1}$ Orlando V. Billoni, ${ }^{1}$ Francisco A. Tamarit, ${ }^{1}$ Dante R. Chialvo, ${ }^{2}$ and Sergio A. Cannas ${ }^{1, *}$ \\ ${ }^{1}$ Instituto de Física de la Facultad de Matemática, Astronomía y Física (IFFAMAF-CONICET), Universidad Nacional de Córdoba, \\ Ciudad Universitaria, 5000 Córdoba, Argentina \\ ${ }^{2}$ Department of Physiology, Northwestern University, Chicago, Illinois 60611, USA
}

(Received 29 September 2008; published 31 August 2009)

\begin{abstract}
Although most networks in nature exhibit complex topologies, the origins of such complexity remain unclear. We propose a general evolutionary mechanism based on global stability. This mechanism is incorporated into a model of a growing network of interacting agents in which each new agent's membership in the network is determined by the agent's effect on the network's global stability. It is shown that out of this stability constraint complex topological properties emerge in a self-organized manner, offering an explanation for their observed ubiquity in biological networks
\end{abstract}

DOI: 10.1103/PhysRevLett.103.108701

PACS numbers: 89.75. Hc, 89.75.Da, 89.75.Fb

Complex networks of interacting agents are ubiquitous, in a wide range of scales, from the microscopic level of genetic, metabolic, and proteins networks to the macroscopic human level of the Internet [1,2]. All of them exhibit high clustering and a relatively short path length compared with random networks. In addition, they frequently show a nonhomogeneous structure, characterized by a degree distribution (the probability of a node to be connected to $k$ other ones) with a broad tail $P(k) \sim k^{-\gamma}$ for large values of $k$, with exponents $\gamma<3$ [1-3]. Several mechanisms have been proposed to give rise to these kinds of topologies $[1,4]$. These mechanisms have successfully explained the origin of complexity in some networks, but it is recognized that another, equally large, number of cases cannot be accounted for by either class of models. In particular, growing biological networks involve the coupling of at least two dynamical processes. The first one concerns the addition of new nodes, attached either during a slow evolutionary (i.e., species lifetime) or a relatively faster developmental (i.e., organism lifetime) process. A second one is the node dynamics which affects and in turn is affected by the growing processes. It is reasonable to expect that the network topologies we finally witness could have emerged out of these coupled processes. This Letter is dedicated to discuss a simple model of this problem, showing that complex networks do emerge under general realistic constraints. It needs to be noted from the outset that the aim of this Letter is not to describe an arbitrary algorithm but to identify a dynamical process able to be implemented by natural systems.

Before introducing the model, and to fix ideas, let us dwell on some concrete general examples. First consider a food web, which is constructed through community assembly rules, strongly influenced by the underlying dynamics of species and specific interactions among them $[5,6]$. Another example could be neuronal networks, where the addition of hundreds of thousands of new neurons is followed by a dynamical process in which neuronal dynamics and connectivity are interrelated in a way not fully understood. Yet a third example at another scale, could be imagined in the context of social networks, in which novice members can be accepted or rejected based on their individual contribution to a global interest, fitness, performance, or profit. In the three examples it is relatively easy to visualize the two processes mentioned above. The consequence of adding a new member with a given connectivity affecting a global instability (stability) is represented in these examples by the abundance (lack) of food, the neuronal welfare (death), or profits going up (down). Notice that each new member may not only result in its own addition or rejection to the system, but it can also promote avalanches of extinctions amongst existing members, an effect we found that strongly influences the network's topology.

Let us consider a system of $n$ interactive agents, whose dynamics is given by a set of differential equations $d \vec{x} / d t=\vec{F}(\vec{x})$, where $\vec{x}$ is an $n$-component vector describing the relevant state variables of each agent and $\vec{F}$ is an arbitrary nonlinear function. One could imagine that $\vec{x}$ in different systems may represent concentrations of some hormones, or the average density populations in a food web, or the concentration of a chemicals in a biochemical network, or the activity of genes in a gene regulation net, etc. We assume that a given agent $i$ interacts only with a limited set of $k_{i}<n$ other agents; thus $F_{i}$ depends only on the variables belonging to that set. This defines the interaction network, as was done previously [7].

We will assume that there are two time scales in the dynamics. On the long time scale (much larger than the observation time), the system is subjected to an external flux (migration, mutation, etc.) of new agents that interact with some of the previous ones and can be incorporated into the system or not, so $n$ (and the whole set of differential equations) can change. On short time scales we assume that $n$ is constant and the dynamics already led the system to a particular stable stationary state $\vec{x}^{*}$ defined by $\vec{F}\left(\vec{x}^{*}\right)=$ 0 [8]. The stability of that solution is determined by the 
eigenvalue with the maximum real part of the Jacobian matrix $a_{i, j} \equiv\left(\frac{\partial F_{i}}{\partial x_{j}}\right)_{x^{*}}$. Therefore, a new agent will be incorporated into the network if its inclusion results in a new stable fixed point, that is, if the values of the interaction matrix $a_{i, j}$ are such that the eigenvalue with maximum real part $\lambda$ of the enlarged Jacobian matrix is negative $(\lambda<0)$. Assuming that isolated agents will reach stable states by themselves after a certain characteristic relaxation time, the diagonal elements of the matrix $a_{i, i}$ are negative and given unity value to further simplify the treatment [7]. The interaction values (i.e., the nondiagonal matrix elements $a_{i, j}$ ) will take random values (both positive and negative) taken from some statistical distribution. In this way we have an unbounded ensemble of systems [7] characterized by a "growing through stability" history. Randomness would be self-generated through the addition of new agents processes. Each specific set of matrix elements after addition defines a particular dynamical system, and the subsequent analysis for time scales between successive migrations is purely deterministic.

These ideas are implemented in a numerical model as follows: At every step the network can either grow or shrink. In each step an attempt is made to add a new node to the existing network, starting from a single agent $(n=1)$. Based on the stability criteria discussed, the attempt can be successful or not. If successful, the agent is accepted, so the existing $n \times n$ matrix grows its size by one column and one row. Otherwise the novate agent will have a probability to be deleted together with some other nodes as further explained below. More specifically, suppose that we have an already created network with $n$ nodes, such that the $n \times n$ associated interaction matrix $a_{i, j}$ is stable. Then, for the attachment of the $n+1$ th node we first choose its degree $k_{n+1}$ randomly between 1 and $n$ with equal probability. Then the new agent interaction with the existing network member $i$ is chosen such that nondiagonal matrix elements $\left(a_{i, n+1}, a_{n+1, i}\right)(i=1, \ldots, n)$ are zero with probability $1-k_{n+1} / n$ and different from zero with probability $k_{n+1} / n$; to each nonzero matrix element we assign a different real random value uniformly distributed in $[-b, b] . b$ determines the interaction range variability, and it is one of the two parameters of the model. Then, we calculate numerically $\lambda$ for the resulting $(n+1) \times(n+1)$ matrix. If $\lambda<0$, the new node is accepted. If $\lambda>0$, it means that the introduction of the new node destabilized the entire system and we will impose that either the new agent is eliminated or it remains but produces the extinction of a certain number of previous existing agents. In order to further simplify the numerical treatment, we will allow up to $q \leq k_{n+1}$ extinctions, taken from the set of $k_{n+1}$ nodes connected to the new one [9]; $q$ is the other parameter of the model. To choose which nodes are to be eliminated, we first select one with equal probability in the set of $k_{n+1}$ and remove it. If the resulting $n \times n$ matrix is stable, we start a new trial; otherwise, another node among the remaining $k_{n+1}-1$ is chosen and removed, repeating the previous procedure. If after $q$ removals the matrix remains unstable, the new node is removed, we return to the original $n \times n$ matrix, and we start a new trial [10].

First we calculated the average connectivity $C(n)$, defined as the fraction of nondiagonal matrix elements different from zero, averaged over different runs. We found that $C(n) \sim n^{-(1+\epsilon)}$ (see supplementary information [11]) for large values of $n$, where the exponent $\epsilon$ depends on $b$ and $q$, taking values $0<\epsilon<1$. Such behavior is characteristic of food webs [12], and it has been interpreted in terms of selforganized criticality concepts [13]; the present results suggest that this is a general behavior in stability-driven selforganized systems.

Next we calculated the degree distribution $P(k)$ of the network with $n=n_{\max }$ for different values of $b$ and $q$. The typical behavior of $P(k)$ is illustrated in Fig. 1 for $b=2$, $q=3$, and different values of $n_{\max }$. We see the emergence of a fat tail $P(k) \sim k^{-\gamma}$ for large values of $n$, with an exponent $\gamma$, independently of the network size (this figure also shows that the drop in the tail of the distribution is a finite size effect). Notice that this relatively small range of the broad tail is what more often is seen in real networks. The qualitative behavior of $P(k)$ for other values of $b$ and $q$ is the same (see supplementary information [11]). The inset of Fig. 1 shows the value of the exponent $\gamma$ as a function of $b$ for different values of $q$. We see that $\gamma$ presents a minimum around $b=2$ for all values of $q$; as $q$ increases the exponent decreases, and for large enough values of $q$ we obtain a nontrivial value of $\gamma<3$ for a broad range of values of $b$.

To exclude the possibility that the observed network topology is trivially associated with a hidden preferential attachment process, we computed the attachment probability $\Pi(k)$, defined as the probability that a new node connects with an already existing node with degree $k$.

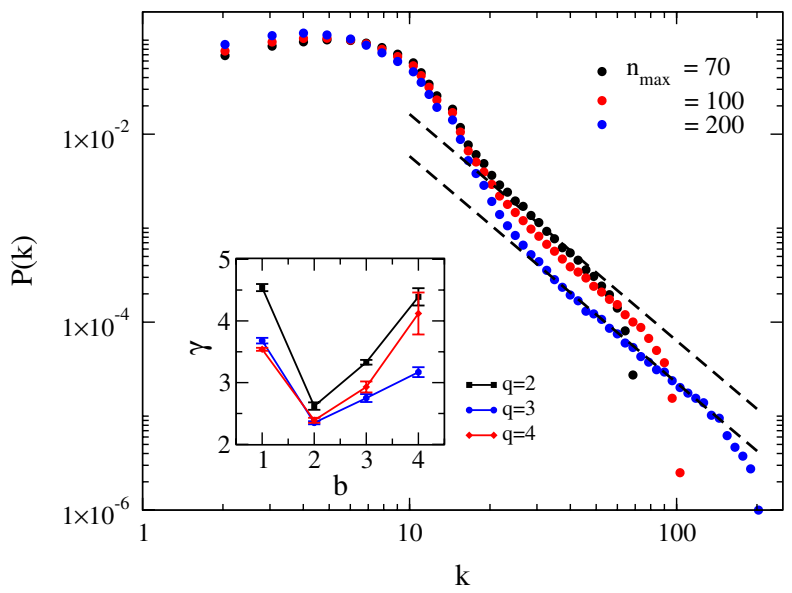

FIG. 1 (color online). Degree distribution $P(k)$ for $b=2, q=$ 3 , and different values of $n_{\max }$; the dashed lines correspond to a power law $P(k) \sim k^{-\gamma}$ with $\gamma \approx 2.4$. The inset shows $\gamma$ as a function of $b$ for different values of $q$. 
Assuming that the average degree $\left\langle k_{i}\right\rangle \ll n$, the attachment probability can be expressed as $\Pi(k)=\sum_{i}^{n_{k}} \Pi_{i}$, where $\Pi_{i}$ is the probability that the new node connects to the already existing node $i, n_{k} \approx n P(k)$ is the number of nodes with degree $k$, and the sum runs over all sites $i$ with degree $k_{i}=$ $k$. If stability selection would favor some kind of preferential attachment mechanism (i.e., if new nodes are attached with larger probability to nodes highly connected), we should expect $\Pi_{i}=k_{i} / \sum_{j=1}^{n} k_{j} \approx \frac{k_{i}}{n(n-1) C(n)}$ and therefore

$$
\Pi(k) \approx \frac{P(k) k}{(n-1) C(n)} .
$$

In Fig. 2 the relative attachment probability $\Pi(k) / P(k)$ in the present model for a fixed network size $n$ and different values of $b$ is compared with the corresponding results for a network of the same size obtained with the BarabásiAlbert (BA) [1] algorithm with connectivity $C(n)$. This quantity shows the expected behavior $\Pi(k) / P(k) \sim k$ for large values of $k$, consistently with Eq. (1). In the present model $\Pi(k) / P(k)$ remains almost constant for a wide range of values of $k$ [including a range of values for which the power law behavior of $P(k)$ has already established] but displays an increasing trend consistent with Eq. (1) for large values of $k$. In other words, in the present model at variance with the BA model, as the network grows, the assembly mechanisms selected by stability show a crossover between two regimes: one dominated by preferential attachment and the other not.

Considering that biological systems are probably never in a completely stable situation, we relaxed the condition of stability $\lambda<0$ and look at networks growing by allowing $\lambda$ to take small positive values so that the characteristic time to leave an unstable fixed point $\tau=\lambda^{-1} \gg 1$. By accepting nodes as long as $\lambda<\Delta$, the calculation of $P(k)$ for different values of $\Delta$ (positive and negative) showed

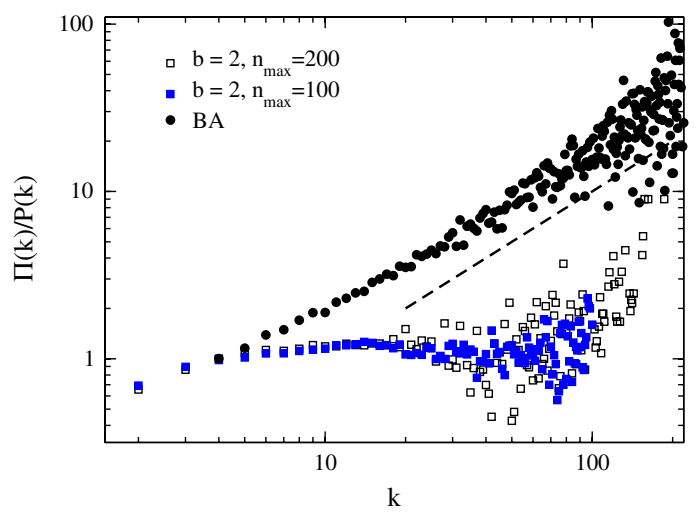

FIG. 2 (color online). Relative attachment probability $\Pi(k) / P(k)$ for different values of $n_{\max }$, compared with the corresponding results for a BA network of the same size. The dashed line corresponds to a linear behavior $\Pi(k) / P(k) \sim k$. similar qualitative behavior, with small variations of the $\gamma$ exponent (see supplementary information [11]).

Next we calculated the average path length $L$ between two nodes and the average cluster coefficient $C c$ for the networks obtained by the present algorithm as a function of the network size $n$. $L$ is defined as the minimum number of links needed to connect any pair of nodes in the network, and $C c$ is defined as the fraction of connections between topological neighbors of any site [1]. In Fig. 3 we show the typical behavior of $L(n)$ and $C c(n)$. We see that $C c(n) \sim$ $n^{-0.75}$ and $L(n) \sim A \ln n+C$. Such scaling behavior is the same one observed in the BA model [1].

As shown in Fig. 3 larger networks becomes less clustered and have a longer minimum path on the average. $L$ and $C c$ are inversely related, as can be seen in the single run plotted in Fig. 3(b). The data correspond to values computed every 50 trials, whether or not the attempt to add a node was successful or not at that trial. In a sense, this is how a natural network would look to an observer if one could take snapshots in time. Clearly both quantities fluctuate in opposite directions, as further shown in the inset,
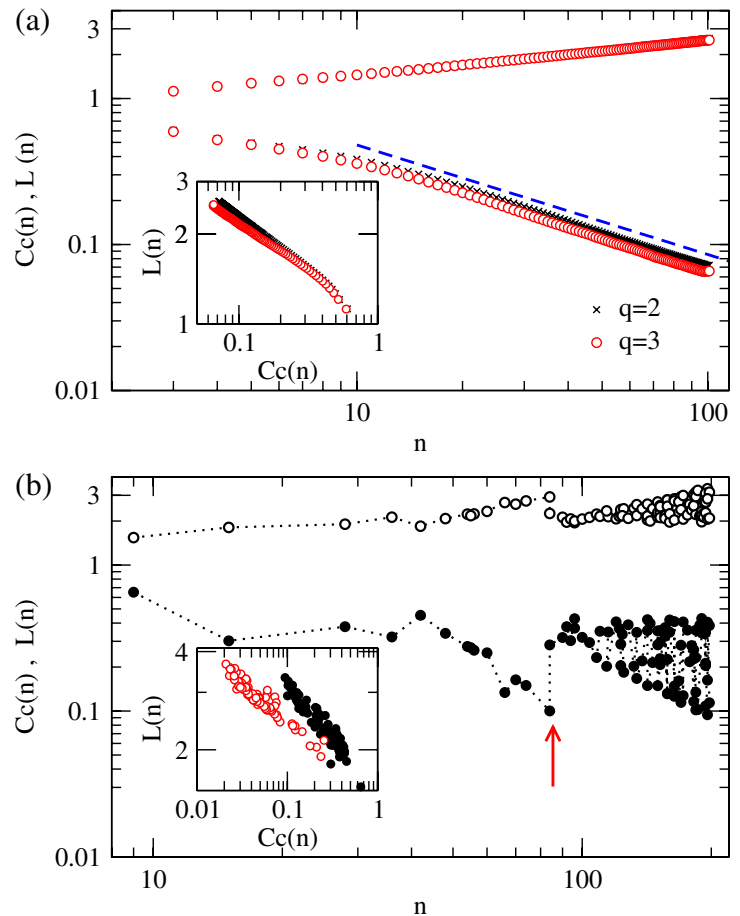

FIG. 3 (color online). (a) Networks' average clustering coefficient $C c(n)$ and $L(n)$ for $b=2$ and different values of $q$ as functions of network size. The dashed line is a guide to the eye corresponding to $C c(n) \sim n^{-0.75}$. The inset shows the same data plotted against each other. (b) $C c(n)$ and $L(n)$ as functions of network size computed from a single network realization. Data are samples taken every 50 trials, regardless of the resulting stability. Notice how fluctuations increase as the network grows. The inset shows the same data plotted against each other (filled circles), in addition to the data computed from a random network with equal size and density of connections (open circles). 


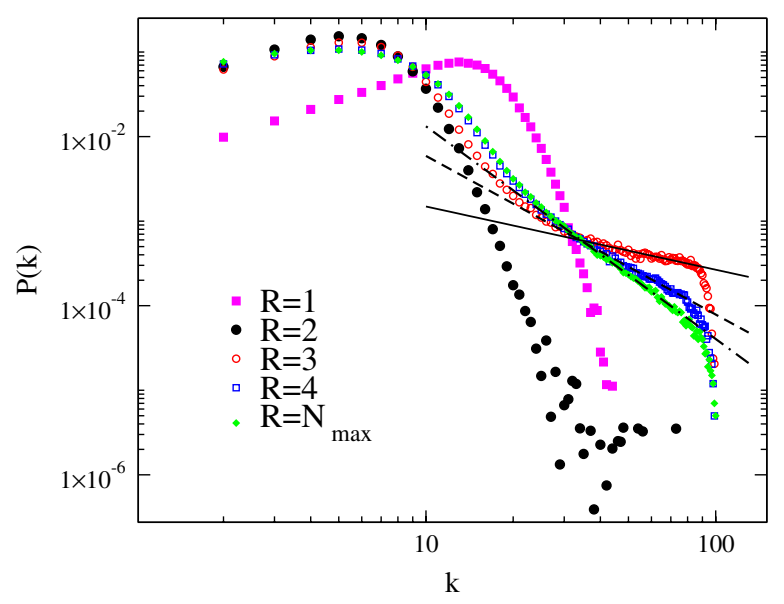

FIG. 4 (color online). Degree distribution $P(k)$ for $b=2, q=$ $3, n_{\max }=100$ and different values of $R$; the straight lines correspond to power law fits.

where the data corresponding to a randomly shuffled network are also plotted for comparison. The behavior of $C c$ and $L$ is linked with the selection dynamics ruling which node is accepted or rejected. The stability constraint favors the nodes with few links, since they modify the matrix $a_{i, j}$ stability much less than new nodes with many links [of course this is reflected in the $P(k)$ density]. Thus, most frequently the network grows at the expense of adding nodes with one or few links, producing an increase of $L$ and a decrease of $C c$. Most of the times, nodes with many links destabilize the network and are rejected, but when one is finally accepted, a large decrease in $L$ together with an increase in $C c$ is observed. This sudden change is the signature of a new network hub, as seen in the example denoted with an arrow in Fig. 3(b). We also verified that those fluctuations lead to a slow diffusivelike growth of the network size $n(t) \sim t^{1 / 2}$ (not shown), where the time is measured in number of trials.

Finally, to consider the effect of local stability selection pressure, we modified the algorithm as follows. Once the new candidate node and its 1 st nearest neighbors (NN) are chosen, we analyze the stability of the subnetwork composed of neighbors up to a range $R(R=1,1 \mathrm{st} \mathrm{NN} ; R=2$, 2nd NN; etc.). In Fig. 4 we show $P(k)$ at $n=n_{\max }$ for different values of $R$. We see that the fat tail $P(k) \sim k^{-\gamma}$ appears as long as $R \geq 3$, which coincides with the value of $L$ for the corresponding net size (see Fig. 3), suggesting a correlation between stability and the self-organized emergence of small world topology. Notice also that considering local stability allows a larger variability in the value of $\gamma(\gamma \approx 0.9$ for $R=3)$, although $\gamma$ quickly converges to the global stability result (for $R>4$ both results are almost indistinguishable).

The robustness of complex networks against error and attack has already been investigated [14] considering the effects of nodes' or links' deletion. The present results show that the consequences of perturbing a single node may depend also on stability, a topic that deserves further clarification. Indeed, closely related results on Boolean networks dynamics already support the generality of this approach [15].

In summary, the analysis of a simple model shows that complex topology can appear in networks as an emergent property driven by a stability selection pressure during the growing process. This suggests yet another explanation for the ubiquity of complex topology observed in different networks in nature.

This work was supported by CONICET, Universidad Nacional de Córdoba, FONCyT Grant No. PICT-2005 33305 (Argentina) and NINDS (USA) Grant No. NS58661.

*cannas@famaf.unc.edu.ar

[1] R. Albert and A.-L. Barabási, Rev. Mod. Phys. 74, 47 (2002).

[2] R. V. Solé, R. Ferrer-Cancho, J. M. Montoya, and S. Valverde, Complexity 8, 20 (2002).

[3] O. Sporns, D. Chialvo, M. Kaiser, and C. Hilgetag, TICS 8, 418 (2004).

[4] M. Baiesi and S.S. Manna, Phys. Rev. E 68, 047103 (2003); B. J. Kim, A. Trusina, P. Minnhagen, and K. Sneppen, Eur. Phys. J. B 43, 369 (2005); S. Laird and H. J. Jensen, Europhys. Lett. 76, 710 (2006).

[5] E. Weiher and P. Keddy, Ecological Assembly Rules (Cambridge University Press, Cambridge, England, 1999).

[6] S. L. Pimm, The Balance of Nature (University of Chicago Press, Chicago, 1991).

[7] R. M. May, Nature (London) 238, 413 (1972).

[8] We consider here only stable fixed points of the dynamics which is, at least in ecology, a common assumption [6]. Nevertheless, the proposed mechanism is expected to work as well for more complex attractors (e.g., limit cycles).

[9] This rule is a bit restrictive, in the sense that one should rather expect an avalanche of extinctions once the system destabilizes. However, this minimal network model is already enough to demonstrate scale free topology.

[10] The process is repeated for $n=n_{\max }$ (typically $n_{\max }=$ 200) and restarted $10^{5}$ times from $n=1$ to obtain statistics of the network's properties for different values of $b$ and $q$.

[11] See EPAPS Document No. E-PRLTAO-103-049937 for additional numerical data of $C(n)$ and $P(k)$ for different values of the model parameters. For more information on EPAPS, see http://www.aip.org/pubservs/epaps.html.

[12] R. V. Solé and B. Goodwin, Signs of Life (Basic Books, New York, 2000).

[13] R. V. Solé, D. Alonso, and A. McKane, Physica (Amsterdam) 286A, 337 (2000).

[14] R. Albert, H. Jeong, and A.-L. Barabási, Nature (London) 406, 378 (2000).

[15] M. Aldana and P. Cluzel, Proc. Natl. Acad. Sci. U.S.A. 100, 8710 (2003). 